\title{
Minimal Set of Constraints for 2D Constrained Delaunay Reconstruction
}

Olivier Devillers — Regina Estkowski — Pierre-Marie Gandoin — Ferran Hurtado —

Pedro Ramos — Vera Sacristán

$\mathbf{N}^{\circ} 4119$

Février 2001

THÈME 2 



\title{
Minimal Set of Constraints for 2D Constrained Delaunay Reconstruction
}

\author{
Olivier Devillers* , Regina Estkowski ${ }^{\dagger}$, Pierre-Marie Gandoin* , Ferran \\ Hurtado $^{\ddagger}$, Pedro Ramos ${ }^{\S}$, Vera Sacristán ${ }^{\ddagger}$ \\ Thème 2 - Génie logiciel \\ et calcul symbolique \\ Projet Prisme
}

Rapport de recherche $n^{\circ} 4119$ - Février 2001 - 9 pages

\begin{abstract}
Given a triangulation $T$ of $n$ points in the plane, we are interested in the minimal set of edges in $T$ such that $T$ can be reconstructed from this set (and the vertices of $T$ ) using constrained Delaunay triangulation. We show that this minimal set consists of the non locally Delaunay edges of $T$, and that its cardinality is less than or equal to $n+i / 2$ (if $i$ is the number of interior points in $T$ ), which is a tight bound.
\end{abstract}

Key-words: Triangulation, Delaunay, 2D, Reconstruction, Minimal Constraints Set 


\section{Ensemble minimal de contraintes pour une reconstruction par Delaunay contraint}

Résumé : Etant donnée une triangulation $T$ de $n$ points du plan, on s'intéresse à l'ensemble minimal d'arêtes de $T$ permettant de reconstruire $T$ en appliquant la triangulation de Delaunay contrainte. On montre que cet ensemble minimal est composé des arêtes de $T$ qui ne sont pas localement de Delaunay, que son cardinal est au plus $n+i / 2$ (où $i$ est le nombre de points intérieurs de $T$ ), et que cette borne est atteinte.

Mots-clés : Triangulation, Delaunay, 2D, Reconstruction, Ensemble minimal de contraintes 


\section{Introduction}

\subsection{Motivations}

In the very active field of geometric compression, the works that deal with meshes compression give generally a method to encode the whole topology of the geometric object $[7,8,2]$. In some cases the topology can be computed from the geometry, for example some terrain models or some finite elements meshes are obtained by using the Delaunay triangulation; in these cases, alternative methods coding only the geometry can be used [6,3] saving the cost of coding the topology. Unfortunately, not all triangulations are Delaunay triangulations, and coding a non Delaunay triangulation must include some topology, although in practice many edges look like Delaunay edges. A method consists in coding only few constrained edges and then reconstruct the topology using the constrained Delaunay triangulation. This idea is exploited in particular by Kim et al. to achieve terrain models compression [4].

This leads to the two theoretical problems that are addressed in Sections 2 and 3:

- given a 2-dimensional triangulation $T$, compute the minimal set of edges $E$ such that $T$ is the constrained Delaunay triangulation of $E$,

- find the theoretical worst-case bound for this minimal set.

We also study the practical efficiency of this approach: we give in Section 4 statistics on the number of non Delaunay edges in some geographic databases.

\subsection{Basic definitions}

Definition 1 (Delaunay criterion) Let $p_{1} p_{2}$ be an edge in a 2-dimensional triangulation $T$. We say that $p_{1} p_{2}$ is a Delaunay edge if there exists a circle going through $p_{1}$ and $p_{2}$ empty of points of $T$ (see Figure 1).

Definition 2 (Local Delaunay criterion) Let $p_{1} p_{2}$ be an edge in $T$, and let $\left\{p_{1} p_{2} p_{3}\right\}$ and $\left\{p_{1} p_{2} p_{4}\right\}$ be the triangles adjacents to $p_{1} p_{2}$. We will say that $p_{1} p_{2}$ is a locally Delaunay edge if the circle $\left(p_{1} p_{2} p_{3}\right)$ does not contain $p_{4}$ or equivalently if the circle $\left(p_{1} p_{2} p_{4}\right)$ does not contain $p_{3}$ (see Figure 2).

Remark 3 In particular, if the quadrilateral $\left\{p_{1} p_{2} p_{3} p_{4}\right\}$ is non convex with reflex angle in $p_{1}$ or $p_{2}$, then $p_{1} p_{2}$ is locally Delaunay (see Figure 3). Convex hull edges can also be considered as locally Delaunay.

\footnotetext{
${ }^{*}$ INRIA, BP93, 06902 Sophia Antipolis, France

†State University of New York at Stony Brook, NY, 11940-3600, USA

$\ddagger$ Univ. Politècnica de Catalunya, Pau Gargallo, 5, 08028 Barcelona, Spain

$\S$ Univ. de Alcalá, Aptdo. de Correos 20, 28871 Alcalá de Henares, Madrid, Spain
} 


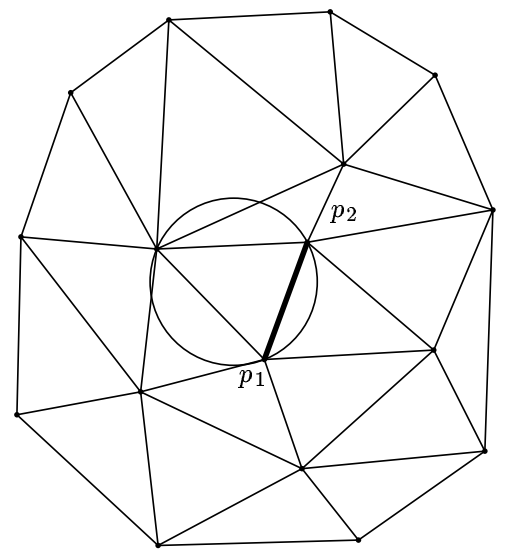

Figure 1: Delaunay criterion

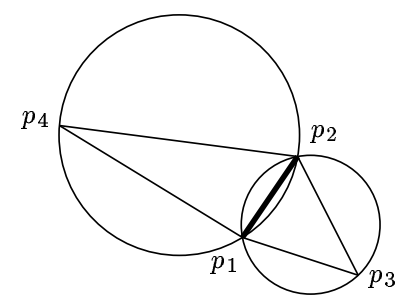

Figure 2: Local Delaunay criterion

$p_{4}$

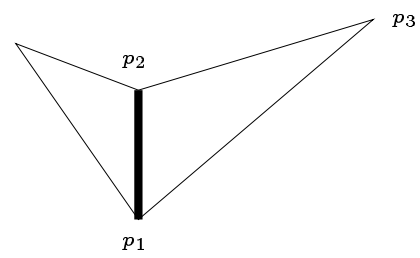

Figure 3: Non convex quadrilateral

Definition 4 (Edge Flip) Let $p_{1} p_{2}$ be an edge in $T$, and let $p_{3}$ and $p_{4}$ be the vertices of its adjacent triangles. We say that $p_{1} p_{2}$ is flipped when it is replaced by $p_{3} p_{4}$ in T. This is possible only if $\left\{p_{1}, p_{2}, p_{3}, p_{4}\right\}$ is a convex quadrilateral (see Figure 4). 

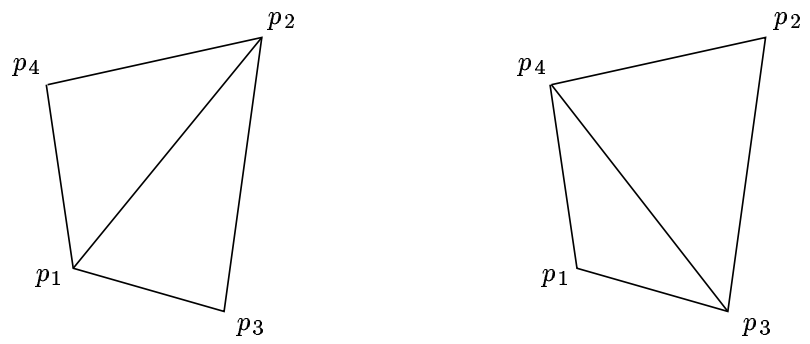

Figure 4: Edge flip

Definition 5 (Constrained Delaunay triangulation) Given a set of points $P$ and a set of edges $E$ in the plane, the constrained Delaunay triangulation $C D(P, E)$ is the unique triangulation such that each of its edges is either in $E$ or locally Delaunay.

Remark 6 This definition is equivalent to the classical definition used for example by Chew [1]: $C D(P, E)$ is the unique triangulation containing $E$ and such that for each remaining edge e of $C D(P, E)$, there exists a circle $c$ with the following properties:

(1) The endpoints of edge e are on the boundary of $c$,

(2) If any vertex $v$ of $E$ is in the interior of $c$ then it cannot be "seen" from at least one of the endpoints of $e$.

This equivalence is shown in particular in an article by Lee and Lin [5].

\section{Minimal set of constraints}

Theorem 7 Let $T$ be a 2-dimensional triangulation, $P_{T}$ the set of its vertices, and $N L D_{T}$ the set of its edges that are not locally Delaunay. Then $N L D_{T}$ is the minimal set $S$ such that $C D\left(P_{T}, S\right)=T$.

Proof: It is easy to see that $C D\left(P_{T}, N L D_{T}\right)=T$. Indeed, $N L D_{T} \subset T$ so we can complete $N L D_{T}$ to obtain $T$. But doing that, we add $T \backslash N L D_{T}$, which, by definition, consists of locally Delaunay edges only. Therefore there is no Delaunay flippable edges and the constrained Delaunay triangulation is over. So $N L D_{T}$ is a sufficient set of constraints.

Reciprocally, let us show that $N L D_{T}$ is necessary. Let $W_{T}$ be a subset of the edges in $T$ such that $C D\left(P_{T}, W_{T}\right)=T$. Let $e$ be an edge in $N L D_{T} \backslash W_{T}$, and $\left\{e_{1}, e_{2}, e_{3}, e_{4}\right\}$ the edges and $\left\{p_{1}, p_{2}, p_{3}, p_{4}\right\}$ the vertices of the corresponding quadrilateral (which is convex by remark 3). Since $C D\left(P_{T}, W_{T}\right)=T$, then $C D\left(P_{T}, W_{T} \cup\left\{e_{1}, e_{2}, e_{3}, e_{4}\right\}\right)=T$. This implies in particular that $e \in C D\left(\left\{p_{1}, p_{2}, p_{3}, p_{4}\right\},\left\{e_{1}, e_{2}, e_{3}, e_{4}\right\}\right)$, which is false since $e$ is non locally Delaunay. Hence such an edge $e$ cannot exist, and $N L D_{T}$ is minimal. 


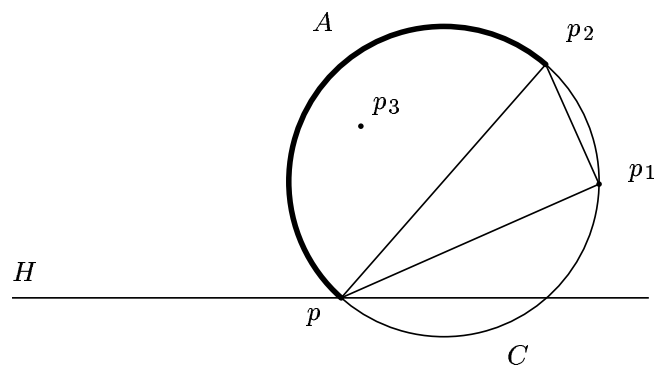

Figure 5: lemma 9

Remark 8 As a direct consequence, we obtain a linear algorithm to compute the minimal set of constraints of a 2-dimensional triangulation.

\section{Worst case study}

In this section, we will show that, given a $n$ points triangulation in the plane, the maximal number of non locally Delaunay edges is half the total number of edges, and that this bound is tight.

Lemma 9 Given a triangulation $T$, any open half-plane $H$ whose boundary contains one interior point $p$ of $T$, contains at least a locally Delaunay edge incident to $p$.

Proof: Let us consider $p p_{1}$, the first edge (going counterclockwise from the boundary of $H$ ) incident to $p$ in $H$ (it exists because $p$ is an interior point of $T$ ). If $p p_{1}$ is the only edge incident to $p$ in $H$, it is necessarily locally Delaunay (this is clear by remark 3 ). Else, let $p p_{2}$ be the second edge incident to $p$ in $H$. Let us assume that none of $p p_{1}$ and $p p_{2}$ is locally Delaunay, and let $C$ be the circle defined by $p, p_{1}$ and $p_{2}$. The $\operatorname{arc} A$ of $C$ bounded by $p$ and $p_{2}$ and not containing $p_{1}$ is necessarily in $H$. Since $p_{2}$ is non locally Delaunay, it exists a point $p_{3}$ lying between the line segment $\left[p, p_{2}\right]$ and the arc $A$ (see Figure 5 ). By iterating the process, we obtain that $p p_{i}$ non locally Delaunay implies $p_{i+1}$ in $H$. Now, since $p$ is an interior point of $T$, there is at least a point $p_{k}$ in the other half-plane. Therefore, the $(k-1)$ th edge - lying in $H$ - is locally Delaunay.

Theorem 10 Let $P$ be a set of $n$ points, $i$ of them being interior. Then every triangulation of $P$ contains at least $n+i / 2$ locally Delaunay edges. Moreover, this bound is tight, up to a constant.

Proof: Lemma 9 clearly implies that for any interior point $p$ of $T$, there are at least 3 edges incident to $p$ in $T$ that are locally Delaunay. When adding the edges on the convex hull, we obtain $\frac{3 i}{2}+(n-i)=n+i / 2$ locally Delaunay edges in $T$. 


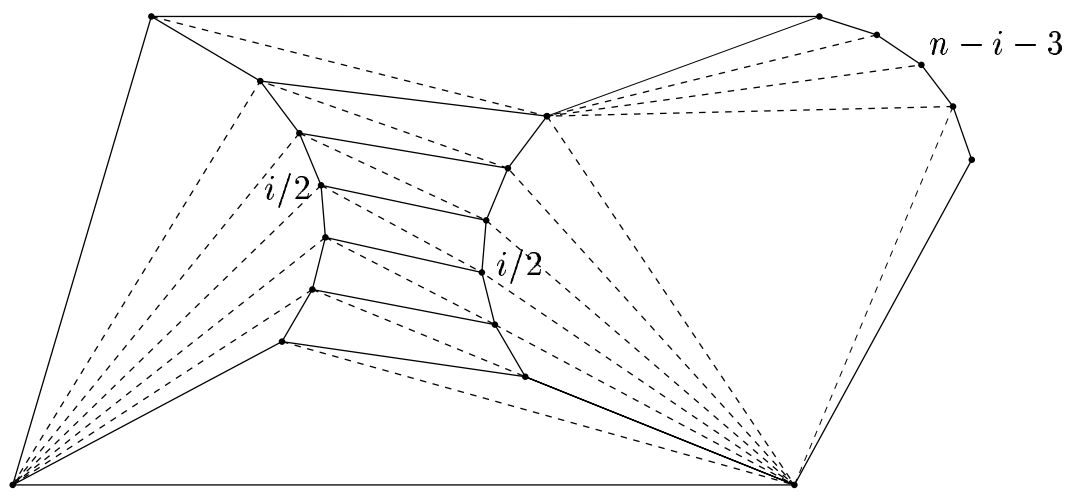

Figure 6: the $n+\frac{i}{2}-5$ non locally Delaunay edges are in dashed lines

As for tightness, since there are $2 n-3+i$ edges in a triangulation, we just showed that any triangulation contains at most $2 n-3+i-(n+i / 2) \approx n+i / 2$ non locally Delaunay edges. The Figure 6 shows a triangulation containing $n+i / 2-5$ non locally Delaunay edges and $n+i / 2+2$ locally Delaunay edges.

\section{Experimental results}

We propose here some statistical results on the size of the set $N L D_{T}$ in practice. The first example of the table comes from the viewpoint collection (http://avalon.viewpoint.com/), whereas the other terrain models tested can be found on a web site of the U.S. Environmental Protection Agency offering several triangulated irregular networks (TIN) in VRML format (http://www.epa.gov/gisvis/vrml/).

Due to the small number of bits used to store the points coordinates, degenerate cases of four cocircular points appears to be frequent. Referring to Definition 2, we call an edge $p_{1} p_{2}$ cocircular if the four points $p_{1} p_{2} p_{3}$ and $p_{4}$ are cocircular. We count in Figure 7 the percentage of non Delaunay edges, and the percentage of cocircular edges. The last two columns of the table show the compression ratios (in bits per vertex) obtained by the algorithm of Devillers and Gandoin [3]. The topologic ratios include the coding of cocircular edges, achieved by an additional sequence of 1 bit per cocircular edge. The geometric ratios correspond to a quantization of 12 bits per coordinate.

\section{Conclusion}

In the information needed to represent a triangulation, we distinguish a geometric part (the vertex positions) and a topological part (the edges). We prove in this paper that if 


\begin{tabular}{|l|c|c|c|c|c|c|}
\hline & $\begin{array}{c}\text { number } \\
\text { of } \\
\text { vertices }\end{array}$ & $\begin{array}{c}\text { number } \\
\text { of } \\
\text { edges }\end{array}$ & $\begin{array}{c}\text { NLD } \\
\text { edges } \\
(\%)\end{array}$ & $\begin{array}{c}\text { cocirc. } \\
\text { edges } \\
(\%)\end{array}$ & $\begin{array}{c}\text { topo. } \\
\text { cost } \\
(\mathrm{bpv})\end{array}$ & $\begin{array}{c}\text { geom. } \\
\text { cost } \\
(\mathrm{bpv})\end{array}$ \\
\hline \hline Crater & 5135 & 14814 & 0.3 & 0.6 & 0.2 & 19.8 \\
\hline Gatlinburg area & 3037 & 9016 & 3.4 & 5.1 & 1.3 & 22.8 \\
\hline Great Smoky Mountains & 7636 & 22736 & 1.0 & 21.4 & 1.1 & 20.4 \\
\hline Mariposa West California & 8390 & 25061 & 0.5 & 18.1 & 0.8 & 19.7 \\
\hline Sevierville/Pigeon Forge & 3516 & 10447 & 3.1 & 9.9 & 1.4 & 21.8 \\
\hline
\end{tabular}

Figure 7: statistics on the minimal constraints set of practical models

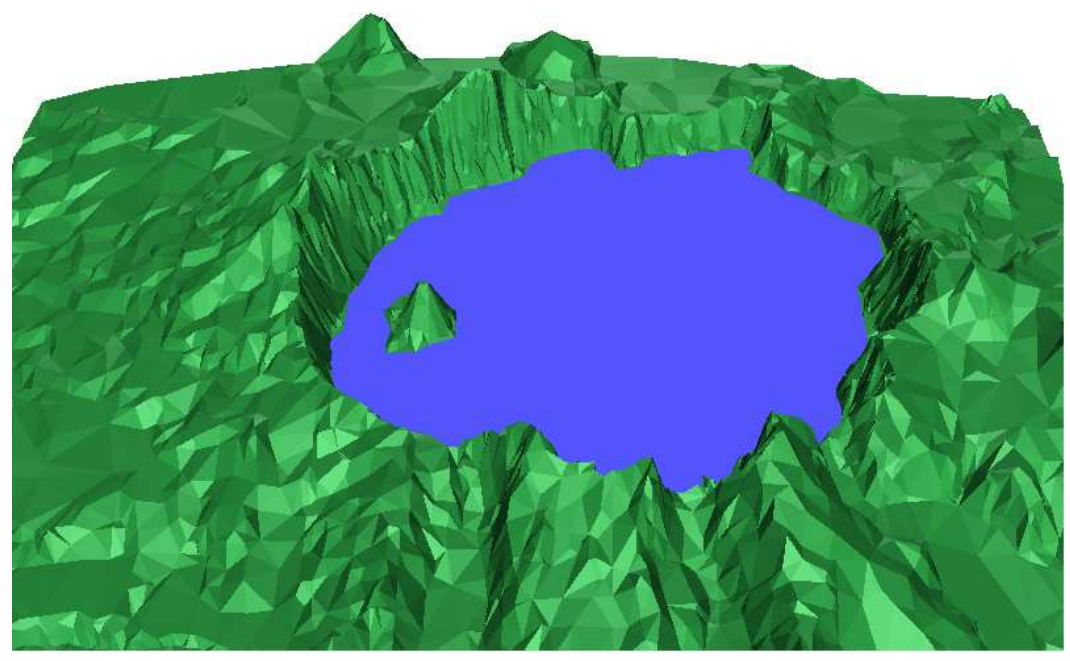

Figure 8: the "crater" terrain model

only the non locally Delaunay edges of a triangulation are stored, then other edges can be reconstructed by constrained Delaunay triangulation. We also prove that this number cannot exceed half of the total number of edges.

Although this bound is tight on some pathological examples, our experiments on real data sets shows a practical rate of non locally Delaunay edges of less than $3 \%$, which yields to a very effective compression of the topological part of the triangulation.

For three-dimensional meshes, the definition of the constrained Delaunay triangulation (definition 5) generalizes straightforwardly. Unlike the $2 D$ case, given a set of constraints, the CDT does not always exist. However, given a triangulation $T(P, E)$ and its non locally Delaunay faces $N L D_{T}, C D\left(P, N L D_{T}\right)$ exists and the theorem 7 generalizes easily. 


\section{Acknowledgements}

Most of this work was made possible by grants from the Picasso French-Spanish collaboration program and the Accion Integrada Francia-España HF99-112. R. Estkowski acknowledges support from the Joint Commission USA-Spain for Scientific and Technological Cooperation, Project 98191. F. Hurtado and V. Sacristán acknowledge additional support from CUR Gen. Cat. 1999SGR00356 and Proyecto DGES-MEC PB98-0933. P. Ramos acknowledges support from the Proyecto DGES-MEC PB98-0713-C02-01 and the Universidad de Alcalá E041 / 2000 .

\section{References}

[1] L. P. Chew. Constrained Delaunay triangulations. Algorithmica, 4:97-108, 1989.

[2] D. Cohen-Or, D. Levin, and O. Remez. Progressive compression of arbitrary triangular meshes. In IEEE Visualization 99 Conference Proc., pages 67-72, 1999.

[3] Olivier Devillers and Pierre-Marie Gandoin. Geometric compression for interactive transmission. In Proc. of IEEE Visualization 2000, 2000.

[4] Y.-S. Kim, D.-G. Park, H.-Y. Jung, and H.-G. Cho. An improved TIN compression using Delaunay triangulation. In Pacific Graphics 99 Conference Proc., October 1999.

[5] D. T. Lee and A. K. Lin. Generalized Delaunay triangulation for planar graphs. Discrete Comput. Geom., 1:201-217, 1986.

[6] J. Snoeyink and M. van Kreveld. Linear-time reconstruction of Delaunay triangulations with applications. In Proc. Annu. European Sympos. Algorithms, number 1284 in Lecture Notes Comput. Sci., pages 459-471. Springer-Verlag, 1997.

[7] G. Taubin and J. Rossignac. Geometric compression through topological surgery. ACM Transactions on Graphics, 17(2), 1998.

[8] C. Touma and C. Gotsman. Triangle mesh compression. In Graphics Interface 98 Conference Proc., pages 26-34, 1998. 


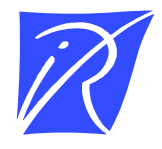

Unité de recherche INRIA Sophia Antipolis 2004, route des Lucioles - B.P. 93 - 06902 Sophia Antipolis Cedex (France)

Unité de recherche INRIA Lorraine : Technopôle de Nancy-Brabois - Campus scientifique 615, rue du Jardin Botanique - B.P. 101 - 54602 Villers lès Nancy Cedex (France)

Unité de recherche INRIA Rennes : IRISA, Campus universitaire de Beaulieu - 35042 Rennes Cedex (France)

Unité de recherche INRIA Rhône-Alpes : 655, avenue de l'Europe - 38330 Montbonnot St Martin (France)

Unité de recherche INRIA Rocquencourt : Domaine de Voluceau - Rocquencourt - B.P. 105 - 78153 Le Chesnay Cedex (France)

Éditeur

INRIA - Domaine de Voluceau - Rocquencourt, B.P. 105 - 78153 Le Chesnay Cedex (France)

http://www.inria.fr

ISSN 0249-6399 